\title{
Measuring the refractive index of a methanol - water mixture according to the wavelength
}

\author{
Nguyen Tien Dung, ${ }^{1}$ Le Canh Trung, ${ }^{1}$ Nguyen Duy Cuong, ${ }^{2}$ Ho Dinh Quang, ${ }^{1}$ Dinh Xuan Khoa, ${ }^{1}$ Nguyen Van Phu, ${ }^{1}$ \\ Chu Van Lanh, ${ }^{1}$ Nguyen Thanh Vinh, ${ }^{1}$ Do Thanh Thuy ${ }^{1}$ and Bui Dinh Thuan ${ }^{* 1}$ \\ ${ }^{1}$ Lab for Photonic Crystal Fiber, Vinh University, 182 Le Duan Street, Vinh City, Viet Nam \\ ${ }^{2}$ Industria University of Vinh, 26 Nguyen Thai Hoc, Vinh City, Vietnam
}

Received October 25, 2020; accepted March 19, 2021; published March 31, 2021

\begin{abstract}
The refractive index of a methanol-water mixture depending on the wavelength at different concentrations was determined by our experimental method using a Michelson interferometer system. A comparative study of Gladstone-Dale, Arago-Biot and Newton relations for predicting the refractive index of a liquid has been carried out to test their validity for the methanol-water mixture with differen concentrations: $30 \%, 40 \%, 50 \%, 60 \%, 80 \%$, and $100 \%$. The comparison shows a good agreement between our experimental results and the results in the expressions studied over a wavelength range approximately from 450 to $850 \mathrm{~nm}$.
\end{abstract}

Accurate knowledge of the refractive index of a mixture has great relevance in theoretical and applied areas of research. In many instances, refractive index data for liquids cannot be found in reference papers and must be measured as needed $[1 \div 5]$. Therefore, a cost- effective method that also provides high accuracy would apply in practice. Measurements of the refractive index of liquid mixtures are essential to determine the composition of binary mixtures, usually for non-ideal mixtures in which direct experimental measurements are made. Most empirical methods for calculating redundant properties are an attempt to explain non-ideality of intermolecular interactions. Finding a small difference in the refractive index of a mixture is often more important than the absolute value of the index itself and these differences cannot be accurately measured by traditional methods [6] There are several techniques for determining the refractive index of liquids. Among them, we often determine the minimum deviation angle of a light ray passing through the liquid contained in a triangular cell, which is often used [7]. This method has been found to be a relatively simple way of obtaining the refractive index of liquid mixtures where high accuracy is not required.

In the present paper a modified Michelson interferometer is described, which has been employed for measuring the refractive indices of liquids $[8 \div 11]$. The aim of this paper is to extend the use of a Michelson interferometer system for measuring directly refractive index of the methanol water mixture of known thickness. We present a wide spectral interferometric technique employing a lowresolution spectrometer for dispersion measurement of $t$ the group refractive indices of liquids over the

*E-mail: thuanbd@ vinhuni.edu.vn wavelength range approximately from 450 to $850 \mathrm{~nm}$. In the next step we compare the measured results with theoretical models, as known in the works $[12 \div 14]$.

The refractive index of a binary mixture is defined by one of the equations: the Gladstone-Dale relation is used for optical analysis of a liquid, for use in fluid dynamics. The relation has also been used to calculate the refractive index. The Gladstone-Dale (G-D) equation for predicting the refractive index of a binary liquid mixture is as follows [12]:

$$
n(\lambda)-1=\left(n_{1}-1\right) \phi_{1}+\left(n_{2}-1\right) \phi_{2} .
$$

Arago-Biot (A-B), assuming volume additively, proposed the following relation for the refractive index of binary liquid mixtures [13]:

$$
n(\lambda)=n_{1} \phi_{1}+n_{2} \phi_{2}
$$

Newton $(\mathrm{N})$ gave the following equation [14]:

$$
\left(n_{m}^{2}(\lambda)-1\right)=\left(n_{1}^{2}-1\right) \phi_{1}+\left(n_{2}^{2}-1\right) \phi_{2}
$$

where, $n_{m}, n_{1}$ and $n_{2}$ are the refractive index of the mixture, refractive indices of pure components 1 and 2 respectively; $\phi_{1}=x_{1} V_{1} / \sum x_{i} V_{i}$ and $\phi_{2}=x_{2} V_{2} / \sum x_{i} V_{i}$ are the volume fraction of pure component 1 and 2 , respectively; $x$ is the mole fraction, $V_{i}$ is the molar volume of component $i$.

The phase refractive index of water as a function of the wavelength will be expressed as the following formula [15]:

$$
n_{1}^{2}(\lambda)=1+\frac{\mathrm{A}_{11} \lambda^{2}}{\lambda^{2}-\mathrm{B}_{11}}+\frac{\mathrm{C}_{11} \lambda^{2}}{\lambda^{2}-\mathrm{D}_{11}}
$$

with parameter values $\mathrm{A}_{11}=0.75831, \mathrm{~B}_{11}=0.01007, \mathrm{C}_{11}$ $=0.08495, \mathrm{D}_{11}=8.91377$.

The phase refractive index of methanol [16]:

$$
n_{2}^{2}(\lambda)=\mathrm{A}_{12}+\mathrm{B}_{12} \lambda^{2}+\frac{\mathrm{C}_{12}}{\lambda^{2}}+\frac{\mathrm{D}_{12}}{\lambda^{4}}+\frac{\mathrm{E}_{12}}{\lambda^{6}},
$$

with parameter values $\mathrm{A}_{12}=1.745946239, \mathrm{~B}_{12}=$ $-0.005362181, \mathrm{C}_{12}=0.004656355, \mathrm{D}_{12}=0.00044714, \mathrm{E}_{12}$ $=-0.000015087$.

The principal diagram of interference with two beams of light is illustrated in Fig. 1. Let $\Delta L\left(\lambda_{0}\right)=L_{0}-l$ be the 
optical path difference between two beams of light in a Michelson interferometer, $L_{0}$ is the initial position of mirror $\mathrm{M}_{2}, t$ is the thickness of a cuvette, $\lambda_{0}$ is the wavelength in which the central fringe and the initial position are coincident (in Fig. 2). When the cuvette has no material medium, the group refractive index of cuvette material $\mathrm{N}\left(\lambda_{0}\right)$ is given by the equation:

$$
N\left(\lambda_{0}\right)=1+\frac{\Delta L\left(\lambda_{0}\right)}{t}
$$

When the cuvette is filled with the liquid of a given refractive index $\mathrm{n}_{l}$, the optical path difference between the two beams of light in the Michelson interferometer is calculated from the following equation:

$$
\Delta_{M}^{\prime}(\lambda)=2\left(L^{\prime}-l\right)-2 t(n-1)-2 d\left(n_{l}-1\right),
$$

where, $L^{\prime}$ is the optical path of the beam after reflection on the $\mathrm{M}_{2}$ mirror (the second branch), $l$ is the optical path of the beam after reflecting on the $M_{1}$ mirror (the first branch), $n$ is the phase refractive index of cuvette material, $d$ is the thickness of the liquid in the cuvette.

When the incident light is white light, the period of fringe spacing measured by the interferometer is calculated as follows:

$$
\Lambda(\lambda)=\frac{\lambda^{2}}{\Delta_{M}^{\prime}},
$$

where $\lambda$ is the wavelength, $\Delta_{M}$ ' the group optical path difference between two interference branches. The group optical path difference displacement of Eq. (9) is written as follows:

$$
\Delta_{M(g)}^{\prime}(\lambda)=2\left(L^{\prime}-l\right)-2 t[N(\lambda)-1]-2 d\left[N_{l}(\lambda)-1\right],
$$

where $N(\lambda)$ is the group refractive index of the cuvette, $N_{l}(\lambda)$ is the group refractive index of methanol-water mixture.

At the position of an equalization wavelength, the optical path difference is equal to zero. Therefore, the position of mirror $\mathrm{M}_{2}$ is determined by the following equation:

$$
L^{\prime}\left(\lambda_{0}\right)=l+t\left[N\left(\lambda_{0}\right)-1\right]+d\left[N_{l}\left(\lambda_{0}\right)-1\right] .
$$

If $\Delta \mathrm{L}^{\prime}\left(\lambda_{0}\right)=\mathrm{L}^{\prime}\left(\lambda_{0}\right)-\mathrm{L}_{0}=\mathrm{L}^{\prime}\left(\lambda_{0}\right)-1$ is the distance between mirror $\mathrm{M}_{2}$ and its initial position, the group refractive index of water is given by the equation:

$$
N_{l}\left(\lambda_{0}\right)=1+\frac{\Delta L^{\prime}\left(\lambda_{0}\right)-t\left[N\left(\lambda_{0}\right)-1\right]}{d} .
$$

Finally, having the group refractive index $N(\lambda)$ of the liquid mixture from the experiment, the phase refractive index $\mathrm{n}(\lambda)$ can be determined by using the equation:

$$
\text { (13): } N(\lambda)=n(\lambda)-\lambda \frac{d n}{d \lambda} \text {. }
$$

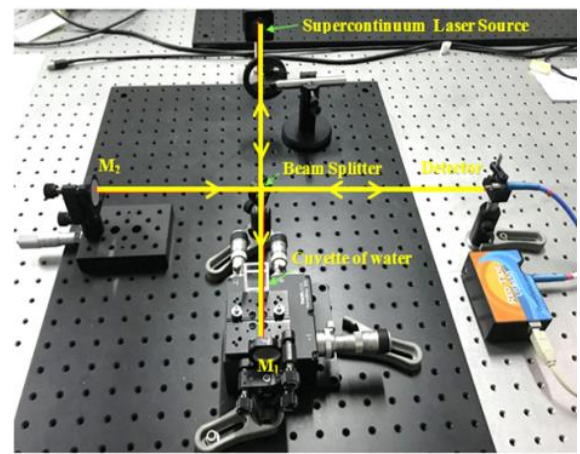

Fig. 1. The schematic of a Michelson interferometer with a supercontinuum laser source.

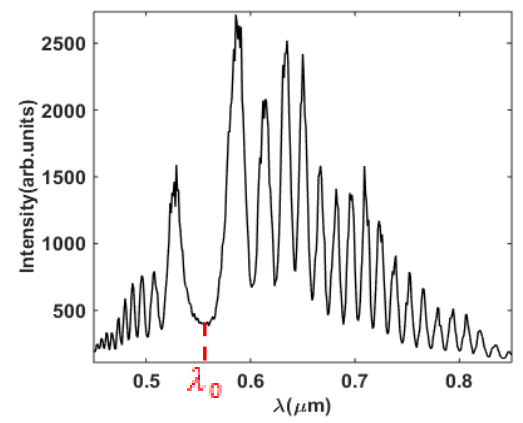

Fig. 2. The spectral interference pattern of the thickness of cuvette $t=2.5 \mathrm{~mm}$ and the thickness of water $d=5.06 \mathrm{~mm}, \lambda_{0}$ is the wavelength in which the central fringe and the initial position are coincident.

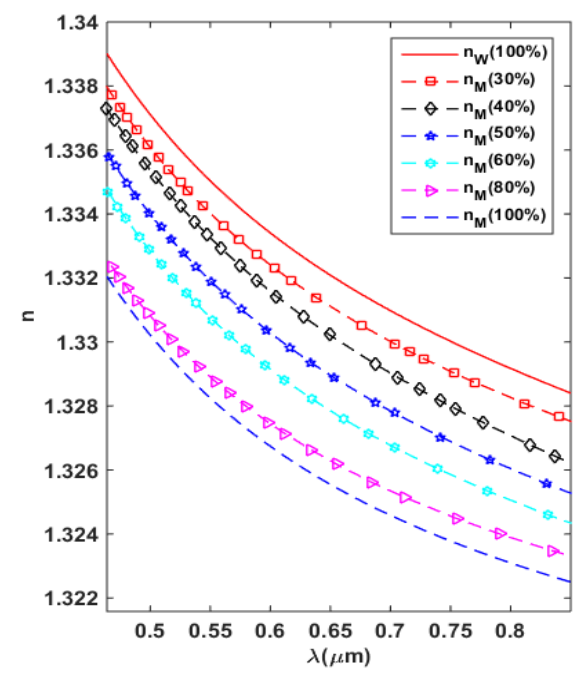

Fig. 3. Phase refractive index of the methanol-water mixture depending on the wavelength at different concentrations.

We conducted the experiments to measure the phase refractive index of a methanol-water mixture with different concentrations 30\%, 40\%, 50\%, 60\%, 80\% and $100 \%$ (w/w), illustrated in Fig.3. The experimental results showed the phase refractive index of the methanol-water mixture as a function of the wavelength in the range of $0.4 \div 0.85 \mu \mathrm{m}$. The phase refractive index of the mixtures has changed between the red line (pure water) and the 
dashed blue line (pure methanol). From Fig.3, it is clear that a percent increase of methanol weight, the phase refractive index of the mixtures is shifted closer to the dashed blue line.

A comparative study of the Gladstone-Dale, AragoBiot and Newton relationships to predict the refractive index of liquid was conducted to test their validity for the methanol-water mixture with different concentrations: $30 \%$ and $60 \%$. The comparison shows a good agreement between our experimental results and the results in the expressions studied over the wavelength range approximately from 0.45 to $0.85 \mu \mathrm{m}$ as in Figure 4 .
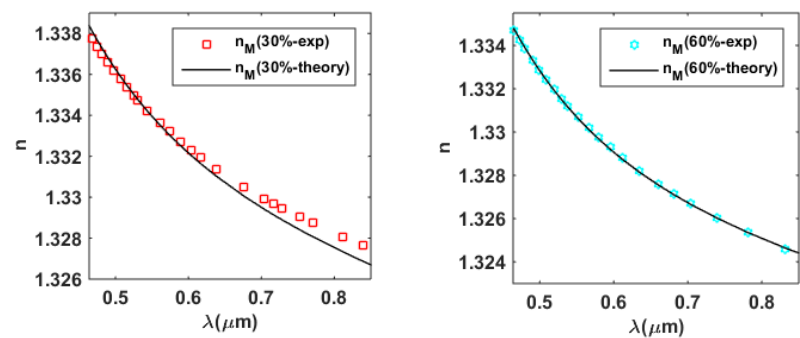

Fig. 4. Phase refractive index of the methanol-water mixture depending on the wavelength at concentrations: $30 \%$ and $60 \%$.

As shown in Fig. 4, the results showed that the differences between the phase refractive index obtained experimentally $n_{\text {exp }}$, and that of the calculated refractive index from the Gladstone-Dale equation $n_{G-D}$ are smallest with $60 \%$ methanol (percent by weight) and largest with $30 \%$ methanol. For more details of the error values are illustrated in Table 1 and Table 2.

Table 1. Experimental and mathematical values of the phase refractive index of a methanol-water mixture at concentration $60 \%$.

\begin{tabular}{llcc}
\hline$\lambda(\mu \mathrm{m})$ & $n_{\text {exp }}$ & $n_{G-D}$ & $\Delta n=n_{\text {exp }}-n_{G-D}$ \\
\hline 0.465 & 1.3346903 & 1.3347787 & -0.0000884 \\
0.473 & 1.3342313 & 1.3342927 & -0.0000613 \\
0.480 & 1.3338482 & 1.3338885 & -0.0000403 \\
0.491 & 1.3332781 & 1.3332899 & -0.0000119 \\
0.499 & 1.3328863 & 1.3328806 & 0.0000057 \\
0.509 & 1.3324213 & 1.3323971 & 0.0000242 \\
0.519 & 1.3319819 & 1.3319424 & 0.0000395 \\
0.530 & 1.3315254 & 1.3314725 & 0.0000529 \\
0.538 & 1.3312097 & 1.3311491 & 0.0000607 \\
0.552 & 1.3306878 & 1.3306170 & 0.0000708 \\
0.566 & 1.3302010 & 1.3301238 & 0.0000772 \\
0.579 & 1.3297773 & 1.3296971 & 0.0000802 \\
0.595 & 1.3292894 & 1.3292087 & 0.0000808 \\
0.612 & 1.3288074 & 1.3287291 & 0.0000783 \\
0.635 & 1.3282072 & 1.3281361 & 0.0000711 \\
0.661 & 1.3275907 & 1.3275317 & 0.0000590 \\
0.682 & 1.3271336 & 1.3270864 & 0.0000472 \\
0.704 & 1.3266883 & 1.3266548 & 0.0000335 \\
0.739 & 1.3260400 & 1.3260298 & 0.0000102 \\
0.781 & 1.3253414 & 1.3253596 & -0.0000182 \\
0.831 & 1.3245970 & 1.3246475 & -0.0000505 \\
0.894 & 1.3237584 & 1.3238443 & -0.0000859 \\
\hline
\end{tabular}

The refractive index of the methanol-water mixture depending on the wavelength at different concentrations was determined by our experimental method using the Michelson interferometer system. From these results, we can blend the mixture of methanol and water into a solution with the desired refractive index by changing the water to methanol ratio. On the other hand, based on these results, we also found that we can check the percentage of methanol-water mixture.

Table 2. Experimental and mathematical values of phase refractive index of methanol-water mixture at concentration $30 \%$.

\begin{tabular}{llcc}
\hline$\lambda(\mu \mathrm{m})$ & $n_{\text {exp }}$ & $n_{G-D}$ & $\Delta n=n_{\text {exp }}-n_{G-D}$ \\
\hline 0.446 & 1.3390844 & 1.339624 & -0.0005398 \\
0.453 & 1.3386348 & 1.339119 & -0.0004838 \\
0.460 & 1.3382053 & 1.338636 & -0.0004306 \\
0.468 & 1.3377374 & 1.338111 & -0.0003732 \\
0.475 & 1.3373468 & 1.337673 & -0.0003257 \\
0.481 & 1.3370251 & 1.337312 & -0.0002869 \\
0.489 & 1.3366139 & 1.336851 & -0.0002376 \\
0.498 & 1.3361737 & 1.336359 & -0.0001853 \\
0.507 & 1.3357557 & 1.335892 & -0.0001361 \\
0.515 & 1.3354014 & 1.335496 & -0.0000947 \\
0.525 & 1.3349796 & 1.335025 & -0.0000458 \\
0.531 & 1.3347370 & 1.334755 & -0.0000179 \\
0.544 & 1.3342366 & 1.334197 & 0.0000393 \\
0.561 & 1.3336292 & 1.333521 & 0.0001080 \\
0.574 & 1.3331968 & 1.333040 & 0.0001565 \\
0.589 & 1.3327288 & 1.332520 & 0.0002086 \\
0.604 & 1.3322905 & 1.332033 & 0.0002572 \\
0.617 & 1.3319323 & 1.331636 & 0.0002967 \\
0.638 & 1.3313912 & 1.331035 & 0.0003565 \\
0.676 & 1.3305127 & 1.330059 & 0.0004541 \\
0.704 & 1.3299333 & 1.329414 & 0.0005196 \\
0.716 & 1.3296999 & 1.329153 & 0.0005464 \\
0.728 & 1.3294744 & 1.328902 & 0.0005726 \\
0.753 & 1.3290277 & 1.328402 & 0.0006257 \\
0.770 & 1.3287397 & 1.328079 & 0.0006609 \\
0.812 & 1.3280748 & 1.327329 & 0.0007461 \\
0.840 & 1.3276622 & 1.32686 & 0.0008025 \\
0.857 & 1.3274217 & 1.326585 & 0.0008370 \\
0.892 & 1.3269467 & 1.326037 & 0.0009093 \\
0.933 & 1.3264191 & 1.325422 & 0.0009971 \\
\hline & & &
\end{tabular}

This work was supported by the Grant number ĐTĐL.CN-32/19.

\section{References}

[1] S. Sharma, P.B. Patel, R.S. Patel, E-J. Chem. 4(3), 343 (2007).

[2] A. Gayathri, T. Venugopal, R. Padmanaban, K. Venkatramanan, R. Vijayalakshmi, IOP Conf. Series: Mat. Scien. Engin. 390, 012116 (2018).

[3] A. Jahan, M.A. Alam, M.A.R. Khan, S. Akhtar, Am. J. Phys. Chem. 7(4), 55 (2018).

[4] N. An, B. Zhuang, M. Li, Y. Lu, Z. Wang, J. Phys. Chem. B 119(33), 10701 (2015).

[5] M. Upadhyay, S.U. Lego, Am. Intern. J. Res. Scien., Techn., Engin. Math. 20(1), 77 (2017).

[6] T.H. Barnes, K. Matsumoto, T. Eiju, K. Matsuda, N. Ooyama, Appl. Opt. 30, 745 (1991)

[7] B.W. Grange, W.H. Stevenson, R. Viskanta, Appl. Opt. 15(4), 858 (1976).

[8] P. Hlubina, Opt. Comm. 193(1-6), 1 (2001)

[9] P. Hlubina, W. Urbanczyk, Meas. Sci. Technol. 16(6), 1267 (2005).

[10] P. Hlubina, D. Ciprian, L. Knyblová, Opt. Comm. 269(1), 8 (2007).

[11] S.R. Kachiraju, D.A. Gregory, Opt. Laser Techn. 44(8), 2361 (2012).

[12] F. Gladstone, D. Dale, Philos. Trans. R. Soc. 148, 887 (1858).

[13] D.F.J. Arago, J.B. Biot, Mem. Acad. Fr. 15, 7 (1806).

[14] S.S. Kurtz, A.L.J. Ward, Franklin Inst. 222, 563 (1936).

[15] K. Moutzouris, M. Papamichael, S. C. Betsis, I. Stavrakas, G. Hloupis, D. Triantis, Appl. Phys. B 116, 617 (2013).

[16] S. Kedenburg, M. Vieweg, T. Gissibl, H. Giessen, Opt. Mater. Expr. 2(11), 1588 (2012). 